\title{
A treatment resistant hypokalemia case associated with leflunomide
}

\author{
Gulali Aktas, Sevim Karacay, Esma Ozmen, Aytekin Alcelik, Ahsen Zeyrek I sci \\ Department of Internal Medicine, Abant Izzet Baysal University Hospital, Bolu, Turkey \\ Correspondence: Gulali Aktas, M.D. Address: Abant Izzet Baysal University, Medical Faculty, Department of Internal \\ Medicine, Bolu, Turkey. Email: draliaktas@yahoo.com
}

Received: December 11, 2013

Accepted: January 16, $2014 \quad$ Online Published: January 20, 2014

DOI : $10.5430 /$ crim.v1n1p10

URL: http://dx.doi.org/10.5430/crim.v1n1p10

\section{Abstract}

Introduction: Leflunomide is a disease-modifying antirheumatic drug which is used in the treatment of rheumatoid arthritis. The most common side effect of leflunomide is diarrhea, however, hypokalemia may also develop during treatment with this drug. Here, we present a case with treatment resistant hypokalemia in a patient who has been on leflunomide treatment for approximately 8 weeks for treatment of rheumatoid arthritis.

Case report: Fifty six years old woman was admitted to internal medicine outpatient clinic with complaints of weakness, constipation, loss of appetite, abnormal weight loss (approximately 10 kilograms) and arthralgias for 3 weeks. Laboratory analyse revealed hypokalemia on admission (serum potassium: $2.3 \mathrm{mmol} / \mathrm{L}$ ). Potassium chloride was infused in isotonic solutions with close monitoring; a potassium value of $4.5 \mathrm{mmol} / \mathrm{L}$ was achieved on the fifth day of treatment. Her complaints such as nausea, constipation, lack of appetite were decreased with normalization of serum potassium.

Conclusion: We suggest close electrolyte monitoring is important if leflunomide treatment was planning especially in patients whose treatment regimens include multiple medications.

\section{Key words}

Leflunomide, Rheumatoid arthritis, Hypokalemia, Inflammation

\section{Introduction}

Leflunomide is a disease-modifying anti-rheumatic drug which is used in the treatment of rheumatoid arthritis ${ }^{[1]}$. It prevents inflammation by inhibition of dihydroorotate dehydrogenase, the enzyme responsible for de novo pyrimidine synthesis ${ }^{[2]}$. The most common side effect of leflunomide is diarrhea. Rash, nausea, vomiting, abdominal pain, liver toxicity, hypocalcemia, hypokalemia, vasculitis are some of the other side effects can be caused by this drug ${ }^{[3-7]}$. Here, we present a case of treatment resistant hypokalemia in a patient receiving leflunomide for approximately 8 weeks for treatment of rheumatoid arthritis. 


\section{Case presentation}

Fifty six years old woman was admitted to internal medicine outpatient clinic with 3 weeks duration of complaints including weakness, constipation, loss of appetite, abnormal weight loss (approximately 10 kilograms) and arthralgias. She has been treated for rheumatoid arthritis and hypertension for fifteen years, also she has been operated for urethral malign neoplasm one year ago. Her medications at the admission time included salazosulfapyridine $2 \times 1000 \mathrm{mg} / \mathrm{day}$, prednisolone $5 \mathrm{mg}$ /day, pantoprazole $40 \mathrm{mg} /$ day, atenolol+chlortalidone 50/12.5/day. Her medical history revealed that she was taking hydroxychloroquine until eight weeks ago when this drug was stopped because of ocular toxicity and leflunomide $10 \mathrm{mg}$ /day was begun at the same time. Physical examination findings were as follows: Body temperature was $36.6^{\circ} \mathrm{C}$, heart rate was 67 beats/minute and blood pressure was 110/60 $\mathrm{mmHg}$. She had joint pain and tenderness on wrists, ankles, elbows and shoulders. Examination of the other systems were normal. The laboratory findings of patients at the time of admission were as follows: serum sodium: $135 \mathrm{mmol} / \mathrm{L}$, potassium: $2.3 \mathrm{mmol} / \mathrm{L}$, urea: $24 \mathrm{mg} / \mathrm{dL}$, creatinine: $0.72 \mathrm{mg} / \mathrm{dL}$, albumine: $4.6 \mathrm{~g} / \mathrm{dL}$, aspartate aminotransferase: $32 \mathrm{U} / \mathrm{L}$, alanine aminotransferase: $28 \mathrm{U} / \mathrm{L}$, erythrocyte sedimentation rate: $51 \mathrm{~mm} / \mathrm{h}$. The $\mathrm{pH}$ was 7.51 on her arterial blood gas analyse. Other laboratory findings were between normal ranges. Her electrocardiogram revealed ST depression and negative T wave on V2-6 derivations. These ECG changes were thought to be due to hypokalemia.

We investigated her past laboratory tests and we revealed that she had had varying degrees of hypokalemia, but no certain etiology had been found. Her minimum serum potassium level was found $3.2 \mathrm{mmol} / \mathrm{L}$ while taking atenolol+chlortalidone. We discontinued leflunomide treatment because of the possible triggering off hypokalemia. Potassium chloride was infused in isotonic solutions according to serum potassium controls. A serum potassium level of $4.5 \mathrm{mmol} / \mathrm{L}$ was achieved on the fifth day of treatment. Her complaints such as nausea, constipation, lack of appetite were decreased with normalization of serum potassium. There was no hypokalemia detected on the follow up for six months of period after she has been discharged from the hospital.

\section{Conclusion}

Different side effects about leflunomide were reported in the literature although limited numbers of these reports ${ }^{[3-7]}$. The most common side effects detected by clinical studies were diarrhea, dyspepsia, nausea, abdominal pain, oral ulcers, impaired liver function tests, increased risks of seconder infections, weight loss and hypertension ${ }^{[8]}$. We have to claim that stopping of Leflunomide and potassium replacement was at the same time prevent to make a concise diagnosis about hypokalemia etiology, but, there was no other reasons for hypokalemia at that time, and her potassium levels were in normal range during follow up after stopping leflunomide. In the lights of these findings, a diagnosis of hypokalemia due to Leflunomide treatment is more likely. Although hypokalemia associated with leflunomide treatment indicated as the drug's side effect, we did not find any severe and resistant hypokalemia case similar to our patient in the literature. In conclusion, we recommend that, close electrolyte monitoring is important if leflunomide treatment was planning especially in patients whose treatment regimens include multiple medications.

\section{References}

[1] Agarwal SK: Biologic agents in rheumatoid arthritis: an update for managed care professionals. Journal of Managed Care Pharmacy. 2011; 17: S14,

[2] Shaw J, Chen B, Wooley P, Huang W-H, Lee A-R, Zeng D: Anti-inflammatory and anti-arthritic effects of a novel leflunomide analogue, UTL-5b (GBL-5b). American journal of biomedical sciences. 2011; 3: 31.

[3] Antonio-Valdiviezo A, Pena-Santos G, Martinez-Torres J: Peripheral neuropathy caused by leflunomide. A case reported with a brief review. Rev Med InstMexSeguro Soc. 2010; 48: 567-570.

[4] Holm EA, Balslev E, Jemec GBE: Vasculitis occurring during leflunomide therapy. Dermatology. 2001; 203: $258-259$. http://dx.doi.org/10.1159/000051761 
[5] Jakob A, Porstmann R, Rompel R: [Skin ulceration after leflunomide treatment in two patients with rheumatoid arthritis]. Journal der Deutschen Dermatologischen Gesellschaft = Journal of the German Society of Dermatology: JDDG. 2006 ; 4: 324-327. http://dx.doi.org/10.1111/j.1610-0387.2006.05934.x

[6] Kamata Y, Nara H, Kamimura T, Haneda K, Iwamoto M, Masuyama J, Okazayi HI, Minota S: Rheumatoid arthritis complicated with acute interstitial pneumonia induced by leflunomide as an adverse reaction. Internal Med. 2004; 43: 1201-1204. http://dx.doi.org/10.2169/internalmedicine.43.1201

[7] Young MG, Rijhsinghani S: Leflunomide and acute hypocalcemia. Endocrine Practice. 2007; 13: 805-807.http://dx.doi.org/10.4158/EP.13.7.805

[8] Osiri M, Shea B, Suarez-Almazor M, Strand V, Wells G, Tugwell P: Leflunomide for the treatment of rheumatoid arthritis: A systematic review and meta-analysis. Arthritis Rheum. 2001; 44: S316-S316. 